\title{
Cluster Analysis on Self-reported Emotional Experiences of Smart TV-viewing
}

\author{
Ilsun Rhiu ${ }^{1}$, Ye Lim Rhie ${ }^{1}$, Gee Won Shin ${ }^{1}$, Myung Hwan Yun ${ }^{1}$ \\ ${ }^{1}$ Department of Industrial Engineering, Seoul National University, Seoul, Korea \\ mhy@snu.ac.kr
}

\begin{abstract}
Identifying users' implicit needs has been recognized as a key factor of product success. Thus, the importance of observing user experience (UX) for comprehensive understanding of users has been continuously emphasized. However, observing user behavior and analyzing collected data require abundant time and effort. Also, there has been little success in developing efficient techniques to assess user experiences. The aim of this paper is to analyze and interpret text data which was collected by user's natural language more effectively and efficiently using SOM (Self-Organizing Map). Diary methods were conducted to collect users' emotional experiences on smart TV by users' natural language with twenty participants for one week. They were especially asked to describe their emotions why they happened in a certain episode. As a result, a total of 311 episodes was collected, and 268 words remained after preprocessing. Based on the co-occurrence matrix, SOM calculated the codebook which represents similarity among episodes. Similarity between episodes could be identified by analyzing a two-dimentional map, and 15 groups were classified. Characteristics of each group could be represented by a frequency of words. From the results of this paper, major patterns of TV-viewing could be identified through SOM. Moreover, implicit user values were identified by analyzing users' emotional experiences, which will help in developing both new and useful functions for the smart TV.
\end{abstract}

Keywords: Emotional experience, Self-Organizing Map, Cluster analysis, Smart TV-viewing, Self-reports

\section{Introduction}

With a growing interest in the topic of User Experience (UX), it became a cornerstone to observe and identify users' latent needs in the product development $[1,2]$. Considering a good user experience is vital to continuous commercial success and it is believed to improve customer loyalty. For example, Jordan [3] suggests that if people have pleasurable experiences with a product, they are more willing to buy the next product from the same company. Although, the notions and necessity of UX have been widely accepted, UX has not yet been enough applied to the process of product development [4]. Thus, it is important to observe a user's behavior to collect and analyze UX for developing new products or services.

Observing UX is complex because the amplitude of experience varies with each interaction, and the overall evaluation of the products changes over time $[5,6]$. Existing observational techniques can be applied to observe instant emotions as well as instrumental systems. Ethnographic study is used to understand users' context of technology usage in real environment, but it has its limitation that it is unable to observe users' perceived emotions. Focus Group Interview (FGI) is usually conducted in the early development procedure as a structured group interview. However, it uncovers priorities and desires, and can be easily influenced by participants. Task analysis is conducted to observe the exact time for each behavior by recording video. Since the experiment is observed in confined environment, it is appropriate for observer to adopt when to know and what the problem is [7]. Since experiential aspects change over time, diary techniques are increasingly used. The diary technique (e.g., self-report, experience sampling method (ESM), day reconstruction method (DRM), etc.) is known to be in an intermediate position of observation between naturalistic settings and controlled settings [8]. It has its benefits in observing the users' experience for the long term and makes researchers able to obtain quantitative as well as qualitative data [9]. Also, a key advantage of a diary method is the short time lag between event occurrence and recording data; hence, there are less subjects' memory lapses and retrospective messages than those of the case with interviews.

However, there are three limitations in the observation techniques. First, subjective bias is incorporated in interpreting the observed data [10]. Also, it is hard to analyze in-depth reasons for their answer [11]. Finally, observing user behavior and analyzing collected data require abundant time and effort [12]. Thus, it is needed to analyze observed data more efficiently and effectively.

To analyze UX more efficiently and effectively, we utilized Self-Organizing Map (SOM) in this paper. The SOM can be used to carry out the classification tasks effectively, especially for the analysis and visualization of a variety of economic, financial, scientific, and manufacturing data sets $[13,14]$. The SOM is a fully connected single-layer linear network, where the output generally is organized in a two-dimensional arrangement of nodes. It is then possible to visually 
identify the clusters from the map. The SOM has the ability to learn and detect regularities and correlations in the inputs, and predict responses from input data [15] The main reason for using SOM rather than other clustering methods is that it would be possible to gain some idea from the structure of the data by observing the map due to the topology preserving nature of the SOM [16]. Also, clustering is performed nonlinearly on the given input data sets. The topologically preserving property allows the SOM applied to document clustering, which results in grouping similar documents together in a cluster and organizing similar clusters close together unlike most other clustering methods [17].

For a case study, we used a diary technique (selfreport method) to collect emotional experiences of smart TV-viewing. Then, SOM is used to group (or summarize) the reports into representative called codebooks to form a two-dimensional grid on a visual map. Also, concept hierarchy clustering is conducted on each group to identify characteristics of each group.

\section{Methods}

$\mathrm{TV}$ is one of the most commonly used everyday products these days. It is proper to conduct a diary technique (self-report method) for observing the smart $\mathrm{TV}$-viewing experiences because there might be a lot of episodes about the TV-viewing experiences during a day. Even though the smart TV has been a big seller, the users do not perceive them as a smart product yet. Therefore, it is necessary to figure out how the smart $\mathrm{TV}$-viewing experiences are different from previous TV-viewing experiences.

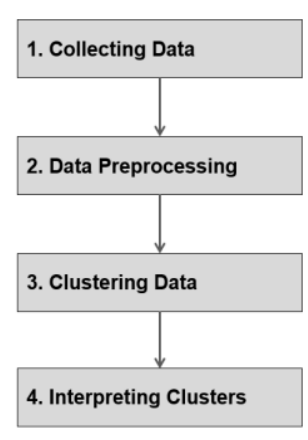

Case study: Diaries on Smart TV

- Sample size: 20 participants

- Observing period: one wee

- Tool: Google template

- Synonym identification

- Stop-words removal

- Stemming and lemmatization

- Transformed into the term-document matrix

- Self-Organizing Map

- Matlab Toolbox v2.1 (Vatanen et al., 2015)

- Observed average of quantization error to identify appropriate map size

- Words Frequency Analysis

- Interpret episodes on close location

Evaluate keywords by frequency analysis

on each episode

Figure 1. The procedure of this study

The detailed process is as follows (Figure 1). First, users' emotional experiences were collected by a diary technique (self-report method). As a result, 20 participants reported 330 episodes for two weeks. Second, to analyze collected data, data preprocessing is required. Synonyms were unified into a representative term, and stop-words were removed. Each sentence was parsed and transformed into a format of term-document matrix, which is becomes a term-episode matrix in this study. Third, clustering analysis was conducted to classify users' emotional experiences. Distances between episodes were visualized by SOM, and intimate ones were grouped into a cluster. Fifteen clusters were observed. Finally, a frequency analysis was conducted for each cluster to interpret clusters which were represented with highly frequent terms. Qualitative interpretation examined more detailed context of use or reasons for emotions.

A self-report method was conducted to collect emotional experiences. The participants recalled their experiences of one day smart TV-viewing and answered the following questions: 'What event occurred', 'How the participant felt', and 'Why the emotions of user's TV-viewing experiences occurred'.

Since various features with high technologies are reflected on the smart $\mathrm{TV}$, it is necessary to recruit participants who are familiar with those kinds of the features. Thus, we recruited participants in ages of twenties and thirties, who might be familiar with the latest electronic products. Thus, the self-report method for observing the smart TV-viewing experience was conducted among 20 participants for a week. The participants were 30.50 years old in average with a standard deviation of 5.65 years. Over a week, a total of 330 complete datasets were retrieved.

The collected transcripts were transformed into the term-document matrix. Synonyms and stop-words were preprocessed and parsed by using automation tool [18], which applies Lucene Korean Analyzer. Sentences on TV program contents were removed except for genre. As a result, total 268 terms were collected.

SOM projects data into a two-dimensional map by an ordered dimensionality-reducing mapping [19]. Each map unit or neuron is represented by its weight of a vector form from the input spaces. By iteratively calculating and comparing distances, similar units are located close. This paper used Toolbox v2.1 [20] and generated 1505 -unit map $(35 * 43)$ with hexagonal grids. The average of quantization error, which is the squared distance between an observed data and its corresponding centroid, was 0.939 .

\section{Results}

To investigate clusters of TV episodes, we analyzed the collected data by the SOM, which is illustrated in Figure 1; the map shows the neurons containing 330 episodes in which similar episodes were mapped onto the similar place. Only considering darker cells indicate a closer relationship, fifteen clusters were evaluated. According to the results of frequency analysis, keywords of episodes were extracted from each cluster (Table 1). Also, the results show that various emotions of user experience were reported.

Episodes in $\mathrm{C} 1$ were related to the context that subjects take a rest while watching TV with family while episodes in $\mathrm{C} 2$ showed subjects being tired and irritated by TV contents. C3 represents episodes that TV viewers were absorbed by TV contents. Group C4 and C5 have much relation with secondary tasks including housework, dining, and conversation. Episodes from C6 to $\mathrm{C} 8$ describe the motivation of watching $\mathrm{TV}$, which are usual interest, cast, and story of drama. Episodes in C9 mainly show positive experience on entertainment programs while those of $\mathrm{C} 10$ are related with negative 
feelings by watching current affairs or documentary. Episodes in $\mathrm{C} 11$ have relation with resting stress by watching TV whereas participants in $\mathrm{C} 12$ watch $\mathrm{TV}$ in an absent sort of way like doing secondary task. C13 and C14 were primarily characterized by positive feelings of subjects, which were affected by TV contents and comfortable environment respectively. C15 mainly considers satisfied emotion because of specification of smart TV including high definition of smart TV and quality of sound.

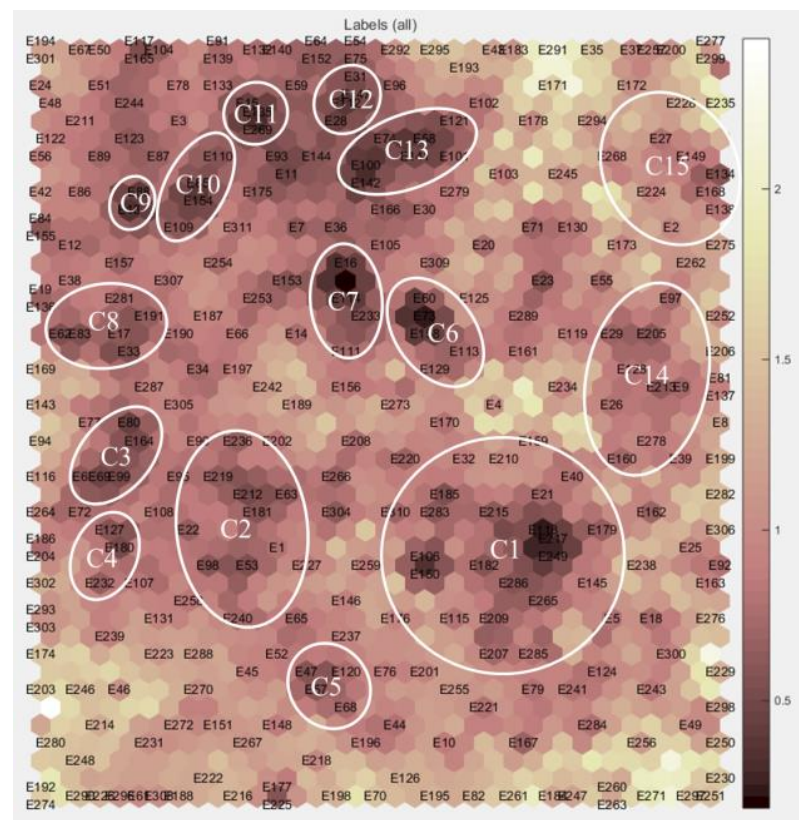

Figure 2. The result of SOM

Table 1 . The result of frequency analysis

\begin{tabular}{c|c|l}
\hline Cluster & $\begin{array}{c}\text { No. of } \\
\text { Episodes }\end{array}$ & \multicolumn{1}{c}{ Keywords } \\
\hline \hline C1 & 19 & Rest, Comfortable, Family \\
\hline C2 & 15 & Tired, Irritated \\
\hline C3 & 5 & Tense, Concentrate, Favorite \\
\hline C4 & 3 & Housework, Concentrate \\
\hline C5 & 4 & Meal, Family, Conversation \\
\hline C6 & 5 & Everyday life, Interest \\
\hline C7 & 5 & Entertainment show, Actor \\
\hline C8 & 6 & Interest, Concentrate, TV Drama \\
\hline C9 & 2 & Interest, Entertainment show \\
\hline C10 & 4 & Anger, Stress, Documentary \\
\hline C11 & 3 & Stress, Rest \\
\hline C12 & 4 & Sound, Hear \\
\hline C13 & 7 & Enjoy \\
\hline C14 & 12 & Comfortable, Rest, Pleasant \\
\hline C15 & 9 & High-definition TV, Satisfy \\
\hline
\end{tabular}

\section{Discussion}

Through utilizing SOM, various contexts of watching TV could be identified, and 15 groups were classified. Episodes in each cluster incorporated various feelings such as comfortable, tired, irritated, tense, interested, angry, stressed, pleasant, enjoyable, and satisfied.

In some clusters of Table 1, there are clusters with similar keywords; episodes in $\mathrm{C} 1$ and $\mathrm{C} 14$ mainly focus on comfort and resting environment, but participants in C1 were usually with their family. Participants in C8 and $\mathrm{C} 9$ were interested in TV contents, but genre was varied. Both $\mathrm{C} 3$ and $\mathrm{C} 4$ mentioned 'concentration'.C3 showed TV viewers who concentrated in TV on the other hands C4 presented viewers couldn't concentrate by housework. $\mathrm{C} 10$ and $\mathrm{C} 11$ were characterized by the term stress. Episodes in $\mathrm{C} 10$ mentioned stresses were occurred by TV contents while C11 presented stresses were relieved by watching TV in a comfort mood. Episodes in $\mathrm{C} 6$ and $\mathrm{C} 8$ presented the motivations of watching TV. In other words, they were interested in the theme in usual and the interest was incurred by story of TV.

By observing classified clusters, various reasons for the emotion were revealed between different clusters. Groups C2, C3, C6, C7, C8, C9, C10, and C13 showed emotion occurred by contents of TV program whereas emotion in $\mathrm{C} 1, \mathrm{C} 2, \mathrm{C} 11, \mathrm{C} 14$ occurred by TV-watching environment. In addition, episodes in $\mathrm{C} 2$ and $\mathrm{C} 15$ revealed emotion was occurred by TV specification and functions, and emotions in $\mathrm{C} 4, \mathrm{C} 5$, and $\mathrm{C} 12$ were dominated by secondary tasks.

By analyzing the two-dimensional map, episodes in close relationships were grouped, but isolated episodes were not counted. Therefore, only major tendencies were identified. Through analyzing the result, it will help developing new functions or services for major consumers. For example, as the result revealed positive emotions were occurred when TV viewers were interested in the TV contents or actors and satisfaction of consumers will increase if TV recommends programs that were related to the areas of interest or actors of whom they are favor That is, new function or service of smart TV could be derived by user-centered approach, rather than solely focusing on usability, UX, or HCI. Therefore, SOM will be a useful tool to observe users' needs in various fields.

\section{Conclusion}

In this paper, we conducted a diary technique (selfreport method) to collect emotional experiences of the smart TV-viewing. Also, we utilized the SOM to analyze the collected emotional experiences data sets efficiently and effectively. As a result, similarity between episodes could be identified by analyzing a two-dimensional map, and 15 groups were classified. Resulted groups helped analyzing major patterns of watching TV enabling user-centered approach.

The SOM-based visualizations offer an intuitive aid for extracting concepts and insights from the collected 
data. For gaining more effective insights, we need information about long-term user experience: how the user's experience with a product evolves over time from the early learning to be a part of daily life. It is a long term user experience that makes people continues to use a product and recommend it to others - not each detail of their individual experiences [21]. In those cases, more data sets will be collected. The principal advantage of the SOM-based display in comparison to such a very large dendrogram is the ability to visualize the entire data set [22]. Also, SOM can be used in conjunction with other clustering methods to visualize clustering results [23], and the hierarchical SOMs could possibly be used to extend results. Thus, the SOM will be more effective to analyze abundant data sets.

Therefore, the results of this study will motivate researchers and practitioners to develop and improve smart TVs and its applications. Also, suggested design implications in this study could be helpful in getting insights under the perspectives of understanding user's implicit needs.

\section{References}

[1] Kraft C.:User Experience Innovation: User Centered Design That Works, A press, 1 edition (January 24), 2012

[2] Kaulio M.A.:Customer, Consumer and User Involvement In Product Development: A Framework and a Review of Selected Methods, Total Quality Management, 9(1): 141-49, 1998

[3] Jordan P.W. :Human factors for pleasure in product use, Applied Ergonomics, 29(1) : 25-33, 1998

[4] Hassenzahl M.:User experience (UX): towards an experiential perspective on product quality, proc. Association Francophone d'Interaction HommeMachine, 11-15, 2008

[5] von Wilamowitz-Moellendorff M., Hassenzahl M. and Platz A. :Dynamics of User Experience: How the Perceived Quality of Mobile Phones Changes over Time, proc. Nordic Conference on Human-Computer Interaction, 74-78, 2006

[6] Karapanos E., John Z., Jodi F. and Jean-Bernard M.:User Experience over Time: An Initial Framework, proc. SIGCHI Conference on Human Factors in Computing Systems, 729-738, 2009

[7] Kuniavsky M.:Observing the user experience: a practitioner's guide to user research, Morgan Kaufmann, 2003

[8] Hyldegård J.:Using Diaries in Group Based Information Behavior Research: A Methodological

Study, proc. Information interaction in context, 151-16, 2006

[9] Kahneman D., Krueger A.B., Schkade D.A., Schwarz N. and Stone A.A.:A Survey Method for Characterizing Daily Life Experience: The Day Reconstruction Method, Science, 306(5702): 1776-1780, 2004

[10] Shapiro D.:The limits of ethnography: combining social sciences for CSCW, proc. the 1994 ACM conference on Computer supported cooperative work,
417-428, 1994

[11] Gable G.G.:Integrating case study and survey research methods: an example in information systems, European Journal of Information Systems, 3(2): 112126, 1994

[12] Lazar J., Feng J.H. and Hochheiser H.:Research methods in human-computer interaction, John Wiley \& Sons, 2010

[13] Petrushin V.A.:Mining rare and frequent events in multi-camera surveillance video using self-organizing maps, proc. the 11th ACM SIGKDD international conference on knowledge discovery in data mining, 794-800, 2005

[14] Wang S.H.:Cluster Analysis using a validated Self organizing method: Cases of problem identification, Intelligent systems in Accounting, Finance and Management, 10(2): 127-138, 2001

[15] Westerlund M.L.:Classification with Kohonen SelfOrganizing Maps, 2005

[16] Alahakoon D., Halgamuge S. and Srinivasan B.:Dynamic self-organizing maps with controlled growth for knowledge discovery. Neural Networks, IEEE Transactions on, 11(3): 601-614, 2000

[17] Gharib T.F., Fouad M.M., Mashat A. and Bidawi I.:Self organizing map-based document clustering using WordNet ontologies, IJCSI International Journal of Computer Science Issues, 9(1): 1694-0814, 2012

[18] Lee M., Rhie Y, Kim J., Lim J.:Development of a Systematic Process and Automation Tool for Semantic Network Analysis on Natural Language, the 15th APIEMS Conference, Jeju, Korea, October 2014 [19] Kohonen T.:The self-organizing map, Proceedings of the IEEE 78.9, 1464-1480, 1990

[20] Vatanen T., Osmala M., Raiko T., Lagus K., SysiAho M., Orešič M., Honkela T., and Lähdesmäki H.:Self-organization and missing values in SOM and GTM, Neurocomputing 147, 60-70, 2015

[21] Kujala S., Roto V., Väänänen-Vainio-Mattila K., Karapanos E. and Sinnelä A.:UX Curve: A method for evaluating long-term user experience, Interacting with Computers, 23(5): 473-483, 2011

[22] Törönen P., Kolehmainen M., Wong G. and Castrén, E.:Analysis of gene expression data using selforganizing maps, FEBS letters, 451(2): 142-146, 1999 [23] Vesanto J. and Alhoniemi E.:Clustering of the selforganizing map. Neural Networks, IEEE Transactions on, 11(3): 586-600, 2000 